\title{
INVESTIGACIONES
}

\section{El buen paciente: un análisis de la relación entre niños y niñas usuarios y profesionales del programa de integración escolar PIE}

\author{
The good patient: an analysis of the relationship between children \\ and professionals of the PIE school integration program
}

\author{
Antonio Moreno Obando ${ }^{a}$, Mónica Peña Ochoa ${ }^{b}$ \\ ${ }^{a}$ Escuela de Psicología. Universidad de las Américas. \\ amorenoo@udla.cl \\ ${ }^{b}$ Facultad de Psicología. Universidad Diego Portales. \\ monica.pena@udp.cl
}

\section{RESUMEN}

Una de las principales políticas que promueven la inclusión escolar en Chile son los Programas de integración escolar (PIE). Estos programas surgen de un modelo de subsidios del estado a escuelas que se entrega a partir de la identificación de necesidades educativas especiales que han sido definidas por diagnósticos basados en el manual DSM-V. Este trabajo se adentra en la relación entre los niños diagnosticados y los profesionales que los tratan dentro de una escuela a partir del concepto de reconocimiento de Axel Honneth. A través de un trabajo cualitativo que incluyó profesionales y niños usuarios, se llegó a resultados como que la relación se basa en un fuerte reconocimiento ideológico donde el diagnóstico de los niños se transforma en principal referente; a la vez que los niños buscan ser buenos pacientes respondiendo lo que los profesionales requieren, en una relación fuertemente enmarcada por el modelo de financiamiento.

Palabras claves: inclusión, integración escolar, escuela, diagnóstico.

\begin{abstract}
One of the main policies that promote school inclusion in Chile is the School Integration Programs (PIE). These programs arise from a state subsidy model for schools that is delivered based on the identification of special educational needs that have been defined by diagnoses based on the DSM-V manual. This work delves into the relationship between diagnosed children and the professionals who treat them within a school based on the concept of recognition of Axel Honneth. Through qualitative work that included professionals and child users, results were reached such that the relationship is based on a strong ideological recognition where the diagnosis of children becomes the main reference; while children seek to be good patients responding to what professionals require, in a relationship strongly framed by the financing model.
\end{abstract}

Key words: inclusion, school integration, school, diagnosis. 
Estudios Pedagógicos XLVI Nº 2: 267-283, 2020

EL BUEN PACIENTE: UN ANÁLISIS DE LA RELACIÓN ENTRE NIÑOS Y NIÑAS USUARIOS Y PROFESIONALES DEL PROGRAMA DE INTEGRACIÓN ESCOLAR PIE

\section{INTRODUCCIÓN}

¿Es posible pensar la inclusión educativa como reconocimiento del otro? Este trabajo toma el concepto de reconocimiento como una relación entre sujetos que fomenta formas de justicia social. Se inserta dentro de aquellos análisis que tratan de comprender el tema de la inclusión en el campo de la justicia social y los derechos humanos. La pregunta se centra en tratar de comprender mejor la relación específica de una de las principales estrategias de trabajo de los llamados Proyectos de Integración Escolar (PIE), la atención individual de niños y niñas por parte de profesionales especialistas como psicólogos y psicopedagogos. Se releva el lugar de niños y niñas que serían los usuarios preferentes del modelo de inclusión. El PIE ha sido criticado -entre varias razones- por estar centrado fundamentalmente en el diagnóstico las llamadas necesidades educativas especiales (NEE). Booth, Ainscow, Black-Hawkins, Vaughan y Shaw (2000) en su "Índice de Inclusión" consideran que las necesidades educativas especiales (NEE) son parte de modelos inclusivos que se centran en los individuos y que no tocan el problema de las barreras a la inclusión que las propias escuelas poseen, es decir, son programas que no cambian las culturas ni las políticas institucionales que pueden reproducir las diferentes formas de exclusión.

Es así como nos pareció necesario mirar lo que pasa con los niños y niñas usuarios de estos programas. Para hacer esto, consideramos el concepto de "inclusión" no como sólo como un concepto operativo de las escuelas, sino que lo situamos en un marco político más amplio, el de la justicia social y el dilema de redistribución-reconocimiento que plantea Fraser (2008). Consideramos que generar cambios en el paradigma de la diferencia como anormalidad a una diferencia como constitutiva de lo social ha sido una dificultad inherente de los modelos educacionales que conservan una mirada de normalización (ver Apablaza, 2017; Matus, Rojas, Guerrero, Herraz, 2018) y funcionan desde una lógica de estandarización de resultados necesaria bajo la modalidad de educación de mercado donde la competencia ha tomado un lugar fundamental como regulador de la calidad de la educación.

Ahora bien, no podemos pensar las prácticas de las escuelas como divorciadas del modelo que las sustenta y guía. En el caso de Chile, uno de los países que financia su educación con un modelo de subvención a la demanda o voucher, el modelo de inclusión que ha propuesto la política pública ha seguido esta lógica, consistiendo en un modelo de subvenciones a casos especiales de estudiantes que se han dividido en dos tipos: estudiantes vulnerables económicamente que no eran incluidos en las escuelas tanto a nivel de acceso escolar, como a nivel de acceso en el aula. La otra subvención especial será para estudiantes con NEE a través de los mencionados PIE, desde el llamado Decreto 170.

Este modelo tiene un efecto en las formas de justicia social. La filósofa feminista Nancy Fraser insiste en que los modelos redistributivos no pueden asumirse como reñidos a los modelos puestos en el reconocimiento. En entornos altamente segregados como el chileno, las luchas no solo se enmarcan en el campo del acceso a derechos y servicios básicos; sino que se trata también de la falta de respeto cultural (Fraser, 2000) y de invisibilización y borramiento de la diferencia. No obstante, es parte de un debate en el que debemos ahondar si es posible considerar redistributivas las políticas de subvenciones especiales. Si bien los modelos de desarrollo impuestos en los años 90's por los organismos económicos internacionales en Latinoamérica consideran que sí (Nina \& Nina, 2004; Mostajo, 2000) también se discute su rol en la repartición justa de la riqueza (Larrañaga, 2007). El dilema que plantea Fraser, no obstante, nos invita como investigadores a preguntarnos justamente 
sobre esto ¿es posible pensar PIE dentro de un marco de justicia social? Y si asumiéramos que estamos frente a una política de carácter redistributivo, ¿es posible pensar que el PIE genera reconocimiento entre los profesionales parte de los PIE y los niños y niñas que son usuarios? ¿y qué tipo de reconocimiento generaría?

Para esto nos basamos en el modelo teórico de Axel Honneth en tanto este filósofo se ha concentrado en dar cuenta que el reconocimiento tiene múltiples formas y también aparece en su forma negativa. Asumimos también que este autor considera que la justicia social se juega en prácticas cotidianas y aspiraciones vitales (Revuelta y Hernández-Arancibia, 2019). Este acercamiento crítico pretende hacer visibles los componentes ideológicos y morales en las relaciones que se establecen entre adultos interventores y niños usuarios de un programa de integración, desde las prescripciones de acciones que especifica la norma del decreto 170 y sus implicancias intersubjetivas. Además de re-enmarcar el modelo desde la teoría del reconocimiento de Honneth, lo que hacemos es pensar metodológicamente la posibilidad de que los niños y niñas que eran usuarias a la fecha puedan dar cuenta de esta relación. Para ello explicamos brevemente qué es PIE, así como se realiza un muy breve marco que conceptualiza la propuesta de Axel Honneth. Luego se muestra el marco metodológico de carácter cualitativo que incluye entrevistas a profesionales del modelo PIE así como a los niños y niñas usuarias a través de un dispositivo lúdico. A continuación de esto, mostraremos el análisis y discusión de los resultados desde el marco analítico que proponemos.

\section{2. ¿QUÉ ES PIE?}

A partir del año 2009 se comienza a implementar el PIE en Chile. La autoridad ministerial lo describe como:

El PIE es una estrategia inclusiva del sistema escolar, que tiene el propósito de contribuir al mejoramiento continuo de la calidad de la educación que se imparte en el establecimiento educacional, favoreciendo la presencia en la sala de clases, la participación y el logro de los objetivos de aprendizaje de todos y cada uno de los estudiantes, especialmente de aquellos que presentan necesidades educativas especiales (MINEDUC, 2009).

El PIE implica fundamentalmente el uso de recursos del Estado en especialistas para el trabajo con estudiantes con necesidades educativas especiales, de manera que presten "ciertos apoyos de tipo extraordinario durante un tiempo específico o durante toda la etapa escolar dependiendo de la evolución de las necesidades educativas especiales y del mejoramiento de las condiciones del contexto escolar" (MINEDUC, 2013).

El marco legal de PIE es el Decreto 170 (2009), el que establece subvenciones estatales para los sostenedores de las escuelas por cada estudiante que cuente con uno de los diagnósticos contemplados en el decreto. Esto implica que mientras más estudiantes con diagnóstico tenga una escuela, mayor cantidad de dinero recibirá, siempre que no sobrepase el límite de 5 estudiantes con diagnósticos asociados a necesidades educativas especiales de tipo Transitorio y 2 estudiantes con diagnósticos asociados a necesidades educativas especiales de tipo Permanente. Según el decreto, se entiende por necesidades educativas especiales de tipo permanente aquellas barreras para aprender y participar que 
Estudios Pedagógicos XLVI Nº 2: 267-283, 2020

EL BUEN PACIENTE: UN ANÁLISIS DE LA RELACIÓN ENTRE NIÑOS Y NIÑAS USUARIOS Y PROFESIONALES DEL PROGRAMA DE INTEGRACIÓN ESCOLAR PIE

determinados estudiantes experimentan durante toda su escolaridad como consecuencia de una discapacidad diagnosticada por un profesional competente, y que demandan al sistema educacional la provisión de apoyos y recursos extraordinarios para asegurar el aprendizaje escolar. Incluye los siguientes déficits o discapacidades: Discapacidad visual, Discapacidad auditiva, Disfasia severa, Trastorno autista y Deficiencia mental severa o multidéficit.

Las necesidades educativas especiales de tipo transitorias, por su parte, son aquellas no permanentes que presentan los alumnos en algún momento de su vida escolar a consecuencia de un trastorno o discapacidad diagnosticada por un profesional competente, y que necesitan de ayudas y apoyos extraordinarios para acceder o progresar en el currículum por un determinado período de su escolarización. Se incluyen las siguientes condiciones: Trastornos específicos del aprendizaje, Dificultades específicas del aprendizaje de la lectura, Dificultades específicas de la lectura y escritura, Dificultad específica del aprendizaje de las matemáticas, Trastornos específicos del lenguaje (TEL), Trastorno déficit atencional con y sin hiperactividad (TDA) o Trastorno hipercinético y Rendimiento en pruebas de coeficiente intelectual (CI) en rango límite, con limitaciones significativas en la conducta adaptativa.

Como se puede apreciar en el listado anterior, en las definiciones conviven al menos tres tipos de NEE: por un lado, las que nacen de la definición psiquiátrica, a través del manual DSM-V como el TDA o el trastorno autista. Por otro lado, están las necesidades orientadas a problemáticas del aprendizaje que son del ámbito de la psicopedagogía. Finalmente, se usan categorías de lo que llamaríamos "discapacidad" y que según Booth, Ainscow et al. (2000) serían definiciones provenientes del campo biomédico tradicional.

El año 2013 se publicó la hasta ahora única evaluación de PIE, que consignó al programa como exitoso en términos de implementación y participación por cantidad de escuelas, pero deficiente en su implementación dentro de las mismas, ya que no logra ser asociado a conceptos como el mejoramiento y la efectividad escolar (Marfán, Castillo, González \& Ferreira, 2013). PIE y su marco legal, el Decreto 170, han sido sujetos de diversas críticas. Peña (2013) considera que el Decreto 170, al concentrarse en el diagnóstico de individuos, sólo regula los aspectos diagnósticos para la selección de los estudiantes que recibirán el financiamiento, sin dar indicaciones acerca de los procesos de integración propiamente tales. Rojas y Armijo (2016) consideran que PIE se verá siempre dificultado por la estandarización de resultados mediante currículo nacional y pruebas estandarizadas, consideradas un contexto adverso para la aplicación de cualquier programa que apunte a la diversidad. Asimismo, señalan que los docentes incorporan dinámicas clasificatorias y estigmatizantes como identificar a los niños como "niño PIE" o "niño SEP", "intensificando formas de nombrar la diversidad estudiantil desde la exclusión” (Rojas \& Armijo, 2016, p. 10). El trabajo disciplinar de los psicólogos PIE ha sido criticado en tanto respondería a un modelo psicoterapéutico de trabajo dentro de la escuela en desmedro del trabajo pedagógico o de otro tipo (Tamayo, Arrau, Sánchez, Besoaín \& Rebolledo, 2018).

\section{EL RECONOCIMIENTO SEGÚN AXEL HONNETH}

En esta investigación nos interesaba trabajar "al interior" de la relación entre los profesionales y los usuarios. Las dinámicas psíquicas y sociales de la interacción al interior de la implementación del PIE fueron entendidas a través de la teoría del reconocimiento de Honneth. Entendemos esto como una filosofía social que continúa el proyecto del 
pensamiento social crítico de la escuela de Frankfurt, que genera en su desarrollo implicancias tanto en la psicología social como en las teorías de las relaciones objetales del psicoanálisis (Honneth, 1997). En estos cruces se genera un fundamento intersubjetivo para pensar el movimiento que hace la conciencia de sí hacia la autonomía, la que se construye desde la tensión moral que se genera entre los sujetos que buscan ser reconocidos. Esta forma elemental de reconocimiento social es señalada por Hegel como eticidad natural donde se distinguen tres estadios: uno educativo o filial donde los sujetos sienten necesidad afectiva del otro y dependen entre sí, otro donde los sujetos se reconocen como propietarios de pretensiones legítimas reguladas por un contrato. Finalmente surge un tercer estadio de solidaridad, en donde los sujetos se intuyen a sí mismos en el otro.

A estas categorías del Hegel de Jena, Honneth incorpora la teoría de la formación de la autoconciencia de la psicología social de George Herbert Mead con la intención de ampliar las posibilidades empíricas de reconocimiento (Honneth, 1997). Los actos que buscan coordinaciones con el otro a través de gestos y palabras generan las interpretaciones sucesivas que diferencian un "yo práctico" conformado por la interiorización de los objetos sociales, de una "consciencia de sí". Como ejemplo del rastreo del propio valor en las respuestas de los otros, Mead se refiere al acto de ejercer derechos, donde suponemos el asentimiento que tiene la comunidad sobre el cuidado de nuestra integridad. (Mead, 2010).

Finalmente, Honneth incorpora en su teoría, conceptos psicodinámicos de la teoría del desarrollo emocional del psicoanalista Donald Winnicott para fundamentar la operación psíquica del reconocimiento. En las observaciones clínicas el psicoanalista describe a la madre como una función que debe sostener e interpretar aquellas manifestaciones primarias del bebé, a pesar de que éste considere estos cuidados como un ataque (Honneth, 1997). Complementando la lectura que hace Honneth de Winnicott, podemos encontrar una alusión directa al desarrollo moral escolar que hace el psicoanalista británico en su artículo "La ética y la educación” de 1963. En él plantea la aparición del desarrollo moral, no a partir de la imposición de un código específico enseñado en la etapa escolar, sino que a través de los intercambios que se producen entre el bebé y la madre. Este intercambio que debe ser suficientemente bueno, por una parte, encuentra aquellas experiencias de satisfacción que conformarán su self, así como también aquellas experiencias frustrantes conformarán una exterioridad, o "no yo" desde el cual, una vez diferenciado, instalar la encrucijada de incorporar o rechazar objetos (Winnicott, 1981).

Considerando esta articulación diversa en la teoría del reconocimiento de Honneth, resulta relevante para comprender el valor constituyente de la subjetividad infantil desde la teoría del reconocimiento, las condiciones del campo intersubjetivo donde se construye el vínculo entre niños y profesionales, especialmente cuando este campo de objetos comunes está predeterminado por la norma en base a aportaciones financieras y acreditaciones de déficit clínicos. Esto, Mayall lo hace notar desde los nuevos estudios de la infancia, donde reconoce el valor teórico de Honneth para comprender cómo valoran los niños sus relaciones con los adultos (Mayall, 2015).

Los patrones de reconocimiento intersubjetivo que Honneth actualiza a partir de las categorías del Hegel de Jena son tres: El primero es el amor, cuyo origen se forja en las relaciones primarias, desde la aquiescencia que se genera entre el bebé y el otro significativo. El segundo es el derecho, como el establecimiento de las obligaciones normativas donde los sujetos sostienen sus relaciones con un Otro generalizado para internalizar las significaciones de su comunidad. El tercero, es la solidaridad como la valoración social de las diferencias 
Estudios Pedagógicos XLVI Nº 2: 267-283, 2020

EL BUEN PACIENTE: UN ANÁLISIS DE LA RELACIÓN ENTRE NIÑOS Y NIÑAS USUARIOS Y PROFESIONALES

DEL PROGRAMA DE INTEGRACIÓN ESCOLAR PIE

personales y particulares de los sujetos y que hace posible un auto-entendimiento cultural. Cuando estos tres tipos de reconocimiento no están presentes en el campo intersubjetivo de la relación, se establece el fenómeno del menosprecio (Honneth, 1997).

En su obra, en "Reconocimiento como Ideología" publicado el 2006, Honneth establece propiedades de un reconocimiento legítimo considerando un posible déficit crítico en la formulación de sus patrones intersubjetivos. Específicamente, hace referencia al cuestionamiento que hace Althusser a la función de confirmación social ideológica y hegemónica del reconocimiento (Honneth, 2006). La referencia de este cuestionamiento aparece en "Ideologías y aparatos ideológicos del estado" de Althusser donde la noción de subjetividad está sostenida por las relaciones de producción del capitalismo cuyo componente ideológico la hace funcional a los medios de producción (Althusser, 1988). Honneth toma esta crítica considerando el efecto del reconocimiento público del cumplimento de las obligaciones, el cual operaría como un mecanismo estandarizado de la ideología hegemónica para el logro de sus fines. Como ejemplo de esta dificultad, pone como ejemplos la figura del esclavo virtuoso (Raeithel, 1995 en Honneth, 1997), la buena madre (Hausen, 1976 en Honneth, 1997) y el soldado heroico (Schilling, 2002, en Honneth, 1997), todos casos en donde el reconocimiento público y la conciencia normativa que han forjado sus identidades, se establecen como una forma más de dominación (Honneth, 2006).

En vista de estas observaciones, Honneth aborda este cuestionamiento estableciendo la diferencia entre un tipo de reconocimiento que genera conformidad, caracterizado como un reconocimiento falso, de carácter ideológico, y otro reconocimiento legítimo, el cual se diferencia por su alcance moral, pero que resulta difícil y necesario identificar en su particularidad a través de evidencias empíricas. Honneth plantea que los reconocimientos ideológicos no son meras coincidencias entre motivaciones legítimas y las operaciones de la dominación, sino expresiones de fundamentos evaluativos con fines específicos.

Para distinguir el reconocimiento legítimo el cual apunta a la autonomía de los sujetos en el campo intersubjetivo, a las anteriores tres categorías del fenómeno del reconocimiento incorpora otras tres características: identificar en el otro cualidades positivas o provistas de valor, que el reconocimiento sea siempre un acto y que ese acto sea producto de una primera intención de reconocer al otro.

En esta misma línea, para distinguir el reconocimiento ideológico, lo define como aquel que como acto genera predisposiciones para cumplir obligaciones basadas en relaciones de dominio. Además, para identificarlo debe por una parte encontrarse un criterio evaluativo proveniente de un juicio externo institucional anterior a la relación intersubjetiva espontánea y por otra una acción retórica expresada en tres características: debe asociar lo positivo al cumplimento de tareas esperadas, deben ser atributos creíbles y que identifiquen a los sujetos con capacidades específicas (Honneth, 2006). En la presente investigación se vuelve un punto crucial no solo identificar patrones de reconocimiento en el campo intersubjetivo del PIE sino que también poder develar su carácter ideológico en caso de estar presente.

\section{MARCO METODOLÓGICO}

El uso de la teoría del reconocimiento de Honneth en estudios de infancia no es nuevo. Thomas (2012) y Thomas, Graham y McDonald (2016) ha propuesto su uso en los temas 
de participación política infantil y para analizar temáticas relacionadas con bienestar en niños y niñas. Este autor indica algo que se repite en nuestra investigación: el modelo de Honneth si bien se basa en el desarrollo infantil a través del trabajo de Donald Winnicott, no toma en cuenta a los niños y niñas como sujetos sociales, necesariamente (Thomas, 2012). Por otra parte, nos hemos basado en el trabajo de Hanhela (2014), para corregir lo que el mismo autor enuncia como el peligro de la psicologización de la teoría honnethiana, es decir, es necesario en el trabajo de análisis mantener el marco político-social donde se desenvuelve el reconocimiento.

El objetivo de la investigación es realizar una observación de campo sobre las relaciones que se producen entre niños y niñas usuarias del programa de integración escolar PIE y los equipos especializados que los atienden en quinto y sexto básico (niños de 10 a 11 años aproximadamente) de un colegio del sector sur-poniente de la Región Metropolitana de Santiago de Chile. Además de enmarcarnos teóricamente en el diseño de la investigación y particularmente en el análisis de la información en la teoría de Honneth, nos enmarcamos en los estudios sociales de la infancia para el diseño de la recogida de datos, asumiendo, como dicen James y Prout (1997) que los niños y niñas son sujeto sociales plenos y no simples sujetos en espera de ser adultos para ejercer sus roles sociales.

La elección del establecimiento educacional se basó en que fuera un colegio mixto, que atendiera población de clase media baja, que recibiera fondos del estado y tuviera en curso un proyecto PIE de al menos 3 años de antigüedad donde hubiese profesionales psicólogos.

La observación se realizó a través de una observación de campo que tuvo una duración de 5 meses y se establecieron cuatro fuentes de información: revisión de documentos, notas de campo, entrevistas a profesionales del equipo PIE y relatos de experiencias lúdicas desde un juego construido especialmente para esta investigación.

Se realizaron 11 entrevistas a los 4 profesionales que conforman el equipo PIE del colegio que realizan clases a los cursos estudiados, además de la jefatura representada por la jefa del programa. Sus relatos confirmaron dos matrices de datos: la primera consistente en aquellas afirmaciones compatibles con las categorías reconocimiento legítimo y reconocimiento ideológico, mientras que la segunda fue la comparación con el texto del Decreto 170 y los normativos propios del colegio cuyas coincidencias fueron insumos para la construcción del material lúdico que levantó el relato de los niños.

Respecto a la construcción del juego, este proceso responde a la necesidad de generar un dispositivo de entrevista más amplio que la entrevista dirigida por un adulto, sobre un tema fuertemente estigmatizante como puede ser el diagnóstico en salud mental. Habíamos llevado a cabo este tipo de metodología en otras investigaciones sobre temas controvertidos con niños y demostró ser una buena forma de acercamiento que no sólo permitía el trabajo con temas complejos sino también permite el trabajo de diálogo grupal. Para la construcción del juego se diferenciaron 66 frases del texto del Decreto 170 que encontraron coincidencias con las afirmaciones realizadas en las entrevistas y notas de campo. El objetivo era poder representar estas aplicaciones normativas de la interacción en una dinámica que permita la libre apropiación de la palabra fuera del común contexto evaluativo en que estos niños usuarios se encuentran.

En el proceso de construcción del juego encontramos 21 afirmaciones de un total 66 frases preseleccionadas del "Decreto 170" y del "Manual de apoyo a la inclusión escolar en el marco de la reforma educacional” (Mineduc, 2016), que cumplían con dos o más 
coincidencias con las afirmaciones realizadas por las profesionales del equipo PIE en las entrevistas. Luego se construyeron cartas con estas afirmaciones, las cuales por un lado estaban expresadas en forma positiva y en el sentido inverso en forma negativa. El mazo con el que se jugó estaba conformado por 42 cartas repartidas en 6 jugadores. Los niños jugaban a recordar experiencias de interacción buscando las cartas que coincidieran con situaciones cotidianas evocadas al inicio de cada turno por los investigadores. Cada jugador debía buscar en el mazo de cartas aquellas que representaran el recuerdo que pudieron evocar, ordenarla al derecho o al revés y esperar su turno para contar el relato de su recuerdo. En la sesión de juego se realizaron tres turnos más una última ronda libre, agrupando sobre la mesa las cartas anteriormente elegidas. En la utilización de las cartas en todas las sesiones del juego, los niños y niñas del programa de ambos cursos usaron el $61 \%$ de las cartas.

Figura 1. Esquema del juego de cartas.

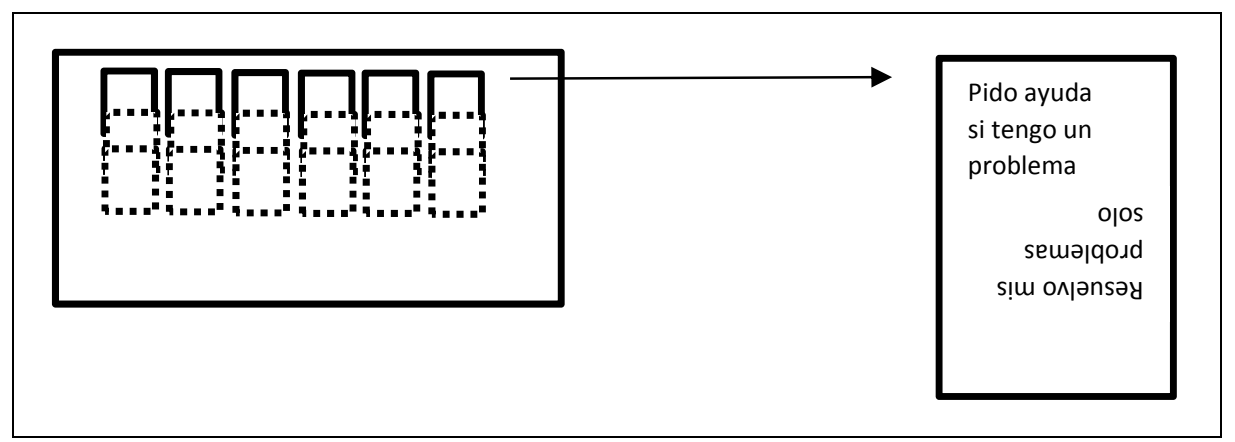

En el trascurso de la investigación se trabajó con 12 niños en dos sesiones diferentes: la primera con 6 niños y niñas usuarias PIE de quinto básico y la segunda con 6 niños y niñas usuarias PIE de sexto básico.

Para analizar la información llevamos a cabo un marco interpretativo basado en las diversas características de la teoría del reconocimiento de Honneth $(1997 ; 2006)$ el que nos permitió pensar desde sus distinciones los hallazgos que aparecieron en el trabajo de campo. El juego como estrategia, que fue construido en el mismo proceso investigativo, facilitó tanto la producción como la interpretación de datos desde un campo donde los niños y niñas participantes interactuaban, tanto con el investigador interventor, como con sus pares vividos y performados en el juego a través del otro.

Las preguntas de fondo con las que organizamos el análisis fueron: ¿Qué tipo de reconocimientos se dan en las interacciones entre equipos técnicos y usuarios del PIE? ¿Será ideológico el reconocimiento que se genera a partir de la identificación al déficit y a la activación de recursos? ¿Cuál es la relación entre la reificación y el reconocimiento ideológico?

El trabajo contó con la revisión de un comité de ética que exigió y revisó el uso de consentimiento informado por parte de los adultos responsables y asentimiento de parte de los niños y niñas participantes. 


\section{ANÁLISIS Y DISCUSIÓN DE LOS RESULTADOS}

\subsection{EL PROFESIONAL COMO INSTRUMENTO DEL PIE Y LA APARICIÓN DEL BUEN PACIENTE}

La primera intensión de los profesionales del establecimiento estudiado es responder a la planificación que se proyecta desde la patología diagnosticada tal como se prescribe en los documentos orientadores de PIE. Por lo tanto, lo que prevalece en la interacción con el usuario es el propósito del profesional PIE de llevar a cabo la actividad preparada, más allá de los contenidos que puedan emerger en la interacción. Los niños son receptores tanto de sus juicios atributivos como de las consecuencias de ellos, sin posibilidad de transformar la interacción. Es tan marcada la predeterminación de la interacción, que los propios usuarios no siempre saben de la intensión de sus tratantes y no parecen presentar -en principiointerés sobre sus reacciones emergentes.

Este aspecto del pronóstico y la planificación aparece de manifiesto en los relatos que realizan los profesionales en donde los déficits registrados en la planificación determinan sus interacciones con los usuarios:

Por ejemplo, mañana tengo en una sala, un niño con Asperger y otro FIL [funcionamiento intelectual limítrofe], entonces tengo dos diagnósticos en un mismo ciclo, una la misma aula y con ellos tengo que saber mezclar (Psicóloga PIE).

Entonces a partir de una evaluación se hace un Plan de Apoyo individual, que no es para todos igual, pero como se trabaja entre todos, porque yo al aula de recursos los traigo todos, trabajo habilidades cognitivas, atención, memoria y algo de materia. Yo trabajo diferentes aspectos con los chiquillos, en algunas ocasiones trabajamos la tolerancia a la frustración dentro de una actividad en particular, la percepción, el autoconcepto, yo los voy tratando de alguna manera ... como se dice.... sin que ellos se den cuenta, pero busco actividades donde pueda introducir todo este tipo de aspectos (Profesora diferencial PIE).

El diagnóstico define la relación en términos que parece cerrarse a otras posibilidades. Las profesionales, por ejemplo, con el caso de un estudiante con diagnóstico Asperger, comentan en las entrevistas sus características de manera muy naturalizada, como si fuera un lenguaje compartido y aceptado en la interacción:

En aula de recursos me ha pasado, principalmente por ejemplo con [niño usuario PIE] aparte de ser Asperger tiene diagnóstico de Oposicionista Desafiante y Déficit Atencional, y toma Aradix [nombre comercial del metilfenidato]. En la mañana lo tengo en aula de recurso en otra jornada, lo que con él no me favorece mucho porque llega sin pastilla. [...] Paramos un rato, con él llegamos a muchos acuerdos; con el Asperger, tú me das esto yo te doy esto otro (Profesora diferencial PIE).

En términos particulares, la profesora diferencial hace explícito el intercambio que realiza en el campo intersubjetivo con el niño que atiende, al que tiene como eje articulador la evaluación previa que se hace de su déficit y la necesidad de la mediación de un objeto de control externo como el medicamento. Desde esta condición se va construyendo en él niño 
un "yo práctico" que responde primero desde un suplemento externo a la relación, que es el medicamento, y que posteriormente responde a una relación rehabilitadora. Efectos de la evaluación previa como determinante de la consciencia de sí, se develan con claridad en el siguiente caso, donde la psicóloga PIE interviene en el quinto básico a propósito de un niño con diagnóstico Asperger:

Entonces, con la educadora de ese curso hicimos un taller donde él [niño con diagnóstico de Asperger] no estaba, entonces conversábamos con ellos de las diferencias que teníamos las personas. Ahí empanzábamos el taller. Les hacíamos ver unas imágenes como percepción visual [...] les preguntábamos cosas en que se reflejaran diferencias, en la en gustos musicales, las cosas por las que nos enojamos [...] Entonces les dijimos: "pero hay otras personas que sentimos que no se parecen en nada a nosotros, que tiene conductas distintas" y ellos empezaron a decir "claro, el S." que es el niño [con Asperger]. Entonces ahí mostramos un video que explica el síndrome y claro, a ellos les calzaban muchas cosas (Profesora diferencial PIE).

El efecto más significativo de esta dinámica de valoración de déficit en el registro y la interacción desde las profesionales es la proliferación de calificativos negativos que nombran y significan los atributos de los sus niños y niñas usuarias, generando que el diagnóstico los configura como personalidades particularmente difíciles para el trabajo y no los dota para la generación de posibles interacciones con el otro, incluyéndolas a ellas:

...Petulante.

Profesora diferencial PIE refiriéndose a niño con diagnóstico de Asperger

Egocéntrico [...] demasiada personalidad.

Profesora diferencial PIE refiriéndose a un niño Asperger

Entonces como es Asperger no lo filtraba, no tenía cuidado, no se adecuaba a los contextos.

Fonoaudióloga PIE

Ellos no sabían trabajar en equipos [...] tiene que ver con las habilidades sociales, más que nada con la flexibilidad mental.

Psicóloga PIE

[los niños que atiendo] son súper inadecuados en muchas ocasiones con el profesor o con el compañero, siempre están metidos en los conflictos en el grupo curso.

Fonoaudióloga PIE

Estos calificativos se constituyen en la interacción como objetos de la comunicación y por lo tanto precursores del "yo práctico" de los usuarios (Mead, 2010) quienes adoptan 
estas reacciones como una forma de reconocer el propio valor de sus reacciones. Según la psicología social de G.H. Mead esta relación de objetos comunicativos genera tanto un diálogo interno entre el "yo práctico" y la consciencia de sí, como la noción del "otro generalizado" que le dará sustento. Para Mead (2010), la experiencia de la persona es una experiencia de la propia acción sobre los otros y de la adopción de la actitud y actuación del otro sobre sí mismo.

Encontramos en las relaciones que propician las profesionales PIE un eje articulador en el reconocimiento de una característica clínica deficitaria, proyectada desde el diagnóstico. Por lo tanto, todo el campo intersubjetivo está determinado por las características patológicas de las niñas y niños usuarios, sin considerar las novedades que puedan surgir desde las interacciones espontáneas. Cualquier componente emergente de la interacción, especialmente si provienen del niño más allá del protocolo que establece su déficit clínico, en caso de ser considerado, pierde importancia. Tan poco valor puede tener el componente emergente que pueda aportar el usuario, que vimos en el campo una intervención psicológica donde no era necesaria la presencia del propio del niño atendido.

Otro caso lo plantea la profesora diferencial del sexto básico, en base a un caso con el que refiere tener una gran cercanía:

Es un niño que dice que toma pastillas faloperas, la que en realidad es Aradix. [...] almorzamos a las 13:00 porque tengo un acuerdo con él, porque, a él le gusta comer mucho y siempre me pregunta por la hora del almuerzo. Entonces yo le paso mi reloj y le digo: "tú me avisas" [...] Por eso he tenido que cambiar un poco mis clases, porque de pronto llega no sé si descompensado pero muy oposicionista, empieza a hablar más fuerte, llama la atención y la clase se va a las "pailas” El siempre me dice “ ¿tú me quieres antes o después de la pastilla?”. Yo cuando puedo lo abrazo. (Profesora diferencial PIE)

En este caso es el propio niño usuario quien hace el señalamiento a la profesora sobre el aspecto deficitario que determina estructuralmente la relación. En su pregunta está señalando al mismo tiempo una reafirmación del vínculo afectivo con la profesional PIE y el reconocimiento de la condición que pone ella para el que el vínculo sea sostenido. Los objetos que se despliegan en la interacción descrita están siempre en torno a las significaciones que emanan del rasgo patológico que esta descrito en la planificación. De esta manera el niño usuario construye una referencia para su "yo práctico" que siempre falla y que debe alterar a través de un tratamiento "falopero" es decir, asociado a la alteración de su estado mental por un poderoso agente externo. El resultado es la incertidumbre que genera el examen de la conciencia de sí, dudando del vínculo, por lo tanto, presionado a adopción de la retórica de la tarea.

Acá no solo vemos la acción del reconocimiento ideológico sometiendo desde su juicio atributivo externo las características de la interacción con su respectivo efecto retórico de exaltación de la tarea y la identidad del déficit, sino que también se aprecia un efecto directo sobre la posición de subyugación psíquica que debe adoptar ante las deficiencias de un yo práctico. El efecto de esta operación es una pregunta por una genuina primera intensión valorativa en el vínculo por parte de la profesora y la adopción obligada de una identidad basada en el buen paciente, por parte del niño usuario. 
Estudios Pedagógicos XLVI Nº 2: 267-283, 2020

EL BUEN PACIENTE: UN ANÁLISIS DE LA RELACIÓN ENTRE NIÑOS Y NIÑAS USUARIOS Y PROFESIONALES

DEL PROGRAMA DE INTEGRACIÓN ESCOLAR PIE

Otro ejemplo de buen paciente ocurre en la siguiente cita:

Pasó que a un niño lo evalué, y él me preguntó, "tía ¿ si me evalúa me voy a quedar con usted?". "Bueno", le dije, "eso depende de si te va bien o te va mal" [...] y me decía "pucha tía debería haber respondido mal... (Psicóloga PIE).

Los aspectos deficitarios que aparecen como objetos de la comunicación conforman un otro generalizado donde sus reacciones son reconocidas como carente de valor en relación con los otros estudiantes y desde los que deben identificarse para poder acceder al reconocimiento de sus derechos como usuarios, por lo tanto, el reconocimiento de derecho se ejerce sólo en tanto hay acceso a la rehabilitación para el desempeño normal a través de un déficit clínico. Por ser reconocido el usuario como deficitario de un logro estándar, la especialización del profesional que lo apoya le hace ser reconocido por la comunidad como aquel que legítimamente puede aspirar a lograrlo a través de la retórica de la superación, más allá si realmente puede lograrlo o no. No puede acreditarse un reconocimiento solidario basado en la singularidad de la persona, aunque los niños traten de mostrarse singulares en el marco rígido del diagnóstico. El diagnóstico no permite que aparezcan esas singularidades y por ello deben adaptarse a ser ese niño que cumple reglamentariamente la prescripción. En los relatos de los profesionales, en documentos y observaciones no se diferencia la incorporación nítida de la particularidad de cada niño o niña usaría del programa más allá de las características patológicas que certifica el diagnóstico que porta.

Este tipo particular de reconocimiento puede desarrollarse sin ser percibido como menosprecio por los usuarios gracias a los actos institucionales que los sitúan en un lugar que probablemente antes tampoco tenían. La política prescrita en el decreto 170 y suscrita como beneficio por el colegio, declara públicamente su primera intensión de reconocer a sus alumnos deficitarios en rendimiento a partir de una evaluación clínica, centrando la legitimidad de la relación desde la predeterminación de la insuficiencia. Si la evaluación es anterior a la relación espontánea de los sujetos que interactúan, se pone en acto el déficit y su rehabilitación desde un criterio homogéneo de rendimiento y cuyo efecto retórico hace a los sujetos construir sus identidades y roles en función de la tarea especial. De esta manera el ejercicio de los propios derechos y la recuperación de una valoración social solo puede hacerse a través de la identificación dicha tarea como una posibilidad de recuperación del valor del aprendizaje perdido por la patología.

\subsection{NIÑOS Y NIÑAS USUARIAS DE PIE: SER POR EL DEBE SER}

Efectivamente la necesidad de mostrarse como el buen paciente aparece de manera constante en los niños y niñas. No obstante, los niños hacen variadas observaciones respecto de ellos mismos, de sus formas de trabajo, de cómo enfrentan las relaciones con los otros, mostrándose menos rígidos que los adultos, pero estos juicios suelen estar supeditados a la función de la tarea escolar que el adulto define:

\section{[Soy] perseverante porque a veces me cuesta entender.}

(Niña usuaria PIE de 5to básico) 
[tengo un] bonito nombre porque soy un .... [dice su nombre alusivo a una figura santa], pero deberían llamarme diablillo porque me porto como un diablillo.

(Niño usuario PIE de 6to básico)

En este mismo sentido, el acto más valorado por los niños y niñas es responder a los requerimientos del profesor más que pensar en sus propias necesidades que sólo son entendidas un paso antes, como déficits y no como la normativa proclama, "necesidades educativas especiales":

Cuando me pierdo, cuando está hablando el profesor, yo trato de entender, trato de seguir para entender. Yo no puedo trabajar con mis compañeros, trabajo sola porque mis compañeros no quieren trabajar conmigo.

(Niña usuaria PIE de 6to básico)

Es posible considerar que la primera intención de los niños y niñas usuarias es la incidencia en la interacción con la profesional. En el caso de los niños y probablemente debido al juego desde el que se tomaron los datos, la interacción se torna fundamental. Si bien la intención es cumplir con el requerimiento de la obligación escolar como acto altamente valorado, en la motivación lo que prevalece es no ser sancionado en la relación que el otro profesor maneja y define:

Yo respondo cuando la profesora me pregunta y yo le digo que sí, pero es solo para que me deje de molestar. En las actividades, cuando sé que la profesora no las va a revisar, yo la dejo y no la hago.

(Niño usuario PIE de 5to básico)

No obstante, apareció otra forma de incidencia en las narraciones producidas por el juego. Un niño narra que un enfrentamiento al profesor sin importar las consecuencias. Si bien no es común en el relato de los niños y niñas situaciones donde pierdan el control en un acto agresivo, sí es posible encontrar la descripción de conflictos sin mediación, donde el sentido de justicia prevalece, el lugar del par compañero se marca y el llamado al profesional a cumplir su función se hace notar. Acá aparece como modo de resolución la eliminación del otro como alternativa:

Yo me acuerdo de la historia que pasó en tercero básico, estábamos haciendo la tarea con mi compañero [...] la tía ayudante [profesora del PIE anterior] que se quedó nos agarró el cuaderno, sacó la hoja la arrugó y las botó, y mi compañero se puso a llorar porque detrás de esa hoja tenía materia importante, y entonces yo lo defendí y me puse a pelear con ella. Empezamos la mansa pelea, yo le dije no puede hacer eso tiene que enseñarle o corregirlo y ella dijo vamos a hablar donde la jefa de UTP [Unidad técnico-pedagógica], yo le dije "vamos donde la Jefa", como yo soy amigo de todo UTP, del director, todo, entonces quedó la mansa embarrada se la llevaron para adentro y a los dos días la echaron.

(Niño usuario PIE de 6to básico) 
Desde los relatos de los niños usuarios, se pueden reafirmar los hallazgos sobre la dinámica del reconocimiento encontrado en el relato de las profesionales del equipo PIE, además de hacer visibles los aspectos ideológicos subyacentes a su identificación a la tarea y la centralidad de su déficit como el núcleo articulador de todas las interacciones. Los objetos que conforman los aspectos subjetivos de los usuarios son siempre reconocidos por un juicio externo, cuya legitimidad está en la institucionalidad que se establece con el diagnóstico proveniente del modelo médico y la razón económica neoliberal que los financia, según la lógica de los PIE definida en el decreto 170. En otras palabras, la actitud práctica de los interactuantes se constituiría en el reconocimiento del déficit individual y la subyacente recapitalización basada en el subsidio que se le entrega a la institución para restituir la pérdida sufrida por la baja competitividad que estos miembros deficitarios generarían.

En segundo lugar, el reconocimiento de estas relaciones se basa en un discurso persuasivo sobre la cualidad positiva de la tarea, la credibilidad de un desempeño cuyo mérito es el esfuerzo y la diferenciación de la capacidad especial/deficitaria. Este efecto retórico genera reconocimientos de atributos deficitarios que financian y dan sentido a la relación rehabilitadora entre niños y niñas usuarias y sus equipos. El propósito general de la implementación del programa como política de gestión educativa en un colegio parece ser, en primera instancia, homogenizar el valor previo de cada estudiante para luego resignificarlo en base a su competencia curricular. El programa PIE le da coherencia al sistema general identificando los desempeños descendidos esperados en cualquier normalidad proyectada, para luego certificarlos como déficits individuales.

Estos aspectos del reconocimiento ideológico tal como nos anunciaba Honneth (2006) encontrados en el reconocimiento de programa PIE, son difíciles de establecer en términos empíricos ya que el carácter evaluativo previo y su efecto retórico podrían reinterpretarse en base a los ajustes de estrategia que cada programa pueda definir en sus procedimientos. Sin embargo, con estos resultados podemos reconocer en el fenómeno de la reificación como un posible indicador del componente ideológico, que en base a su operación económica y subjetiva pudiera establecer una condición verificable de las características del reconocimiento ideológico que genera el programa PIE.

Un elemento diferenciador que aparece con claridad es la fetichización del logro de aprendizaje en el PIE, el cual se refrenda en el valor financiero del déficit individual. El diagnóstico y la intervención rehabilitadora fetichiza el déficit, en su operación le restituye un valor frente a la pérdida que implica un grupo de bajo desempeño a los activos que capitaliza la institución escolar. Desde la operación política de asociar capitalización organizacional con la externalización del déficit, se proyecta una trayectoria escolar que debe ser atendida por la intervención especializada, para de esta manera evitar la descapitalización de un sistema escolar. Esta operación económica cumple todos los criterios de la reificación: pone a los niños usuarios y equipos en una relación mercantil y fetichiza el resultado de la evaluación.

\section{CONCLUSIONES}

Hemos visto como la sociedad chilena ha intentado movilizar sus cimientos culturales, a través de herramientas políticas ciudadanas como las grandes manifestaciones o las tomas de colegios hace más de 10 años (el Movimiento Pingüino, ver Aguilera, 2014), con la 
finalidad de encontrar nuevos espacios normativos donde podamos coordinar nuestras diversidades de manera diferente. De hecho, mientras se redacta esta conclusión, nuestro país cae en la crisis institucional y política más importante de los últimos años. Sus causas y efectos serán materias de numerosos estudios que podrán tomar perspectiva de las implicancias, pero que desde ya se nos aparece como una enorme evidencia la fisura estructural de la instalación de un modelo económico neoliberal instalado en dictadura que ha pretendido dotar de su particular razón económica a cada uno de los aspectos de nuestra vida ciudadana. La vivencia del modelo como injusta es una queja generalizada por lo que es tan necesario pensar las formas de inclusión en la escuela como justicia social.

Sin embargo, una estrategia inclusiva como el Decreto 170, corre el constante peligro para equipos y niños y niñas usuarios de funcionar desde la condición de juicios externos, fetichizaciones de los resultados y operaciones retóricas, otorgándoles valor a los niños y niñas desde pronósticos descontextualizados, especializaciones solo complementarias y lo más importante, construyendo identidades desde el déficit, en donde cada niño usuario debe generar su propio diálogo interno y consciencia de sí, pero ya no en espacios de reconocimiento legítimo de su singularidad y su ciudadanía sino a partir de desempeños parciales que generan conclusiones generales.

Otro elemento a tener en cuenta es el efecto persuasivo del valor del déficit también se expresa en la valoración externa sobre los profesionales del equipo PIE como "especializado". El problema de la psicologización de la pedagogía acá se hace evidente. El valor profesional de PIE se genera en base a sus capacidades complementarias, lo que no permite ampliar el alcance de su intervención. Lo que surge desde estas condiciones es un relato de superación híper especializada que predetermina atributos y generaliza logros esperados en los agentes de la interacción, que es poco realista para entender el desempeño de niños y niñas en el aula. Se establece así un modo retórico de avance y retroceso en la rehabilitación muy difícil de desarticular y de poner en el campo de los pedagógico. La noción de la inclusión como un marco fundamental de justicia social está lejos de aparecer en su formato retórico en tanto el modelo de inclusión que tenemos se centra en individuos o incluso en partes de los individuos en el pequeño panorama del logro de la tarea. Es interesante ver que tampoco pasa, como diría Rojas (2018) a una noción de la inclusión como lo moralmente correcto, tal es el peso de la tarea que el modelo impone.

Es necesario afirmar que la idea de un aprendizaje globalizado y estándar posible de ser comparable no es otra cosa sino una operación retórica e ideológica. Por esto, y en el contexto de la inclusión escolar, es necesario abrir la discusión para que otras nociones de aprendizaje puedan disputar su sentido en la institución escolar. En otras palabras, el reconocimiento ideológico de un niño PIE como de un buen paciente, nos empuja necesariamente a descentrar los juicios externos, apodícticos, que determinan lo que un niño con un diagnóstico determinado debe trabajar pedagógicamente y mirar, necesariamente, el campo político del encuentro con el otro diverso y complejo, con saberes y cultura propia.

Finalmente, estos resultados nos abren preguntas también hacia el ámbito disciplinar ¿cuál es el papel de la psicología en la construcción de nuevos marcos intersubjetivos para la colaboración entre niños y adultos? En particular, la capacidad crítica de la teoría de Honneth, aparece como una posibilidad de convergencia entre los estudios sobre los problemas políticos del poder y aquellos que indagan la capacidad intersubjetiva de los sujetos para coordinarse en acciones comunes. El propio autor señala esta necesidad de incorporar datos empíricos para detectar oportunamente dinámicas del reconocimiento no 
Estudios Pedagógicos XLVI Nº 2: 267-283, 2020

EL BUEN PACIENTE: UN ANÁLISIS DE LA RELACIÓN ENTRE NIÑOS Y NIÑAS USUARIOS Y PROFESIONALES DEL PROGRAMA DE INTEGRACIÓN ESCOLAR PIE

legítimos, para así evitar el peligro de caer en una posición ideológica y acrítica. Este cruce honnethiano que bien puede delimitar un campo posible para la investigación de la psicología social crítica es también una posibilidad de ampliar la investigación en la psicología educacional, la psicología clínica de la infancia y la política pública.

\section{REFERENCIAS BIBLIOGRÁFICAS}

Aguilera, O. (2014). Generaciones. Buenos Aires: Clacso.

Apablaza Santis, M. (2017). Prácticas 'Psi' en el espacio escolar: Nuevas formas de subjetivación de las diferencias. Psicoperspectivas, 16(3), 52-63. Recuperado desde https://dx.org/10.5027/ psicoperspectivas-vpl16-issue3-fulltext-1063

Althusser, L. (1988). Ideología y aparatos ideológicos del Estado, Freud y Lacan. Buenos Aires: Editorial Nueva Visión.

Booth, T. Ainscow, M., Black-Hawkins, K., Vaughan, M. \& Shaw, L. (2000). Índice de Inclusión. Desarrollando el aprendizaje y la participación en las escuelas. Bristol: Centre for Studies on Inclusive Education (CSIE).

Decreto con toma de razón $N^{\circ} 170$. (2009). Fija normas para determinar los alumnos con necesidades educativas especiales que serán beneficiarios de las subvenciones para educación especial. Santiago, Chile: Gobierno de Chile.

Fraser, N. (2008) La justicia social en la era de la política de identidad: redistribución, reconocimiento y participación. Revista de trabajo, 4(6), 83 - 99.

Fraser, N. (2000). ¿De la redistribución al reconocimiento? Dilemas de la justicia en la era «postsocialista» en Fraser, N.; Butler, J. (2000). Redistribución o reconocimiento, un debate entre marxismo y feminismo (pp. 7 -23). Madrid: Traficantes de sueños.

Honneth, A. (2007). Reificación, un estudio en la teoría del reconocimiento. Buenos Aires: Ediciones Katz.

. (2006). El Reconocimiento como ideología. Revista Isegoría, (35),192-150.

(1997). La lucha por el reconocimiento, por una gramática moral de los conflictos sociales. Barcelona: Ediciones Crítica.

Hanhela, T. (2014). Axel Honneth's idea of a drawn-out process of education. Pedagogy, Culture \& Society, 22(3), 369-388, DOI: 10.1080/14681366.2014.891642

James, A. \& Prout, A. (1997). Constructing and reconstructing childhood: contemporary issues in the sociological study of childhood. London: Falmer Press.

Marfán, J., Castillo, P., González, R. y Ferreira, I., (2013). Análisis de la implementación de los programas de integración escolar (PIE) en establecimientos que han incorporado estudiantes con necesidades educativas especiales transitorias (NEET). Chile: CEPE Fundación Chile.

Matus-Cánovas, C.; Rojas-Lasch, C.; Guerrero-Morales, P. y Herraz-Mardones, P. C. (2018). Diferencia y Normalidad: producción etnográfica e intervención en escuelas. Magis, Revista Internacional de Investigación en Educación, 11(23), 23-38. doi: 10.11144/Javeriana.m11-23. dnpe

Mayall, B. (2015). Understanding inter-generational relations: the case of health maintenance by children. Sociol Health Illn, 37(2), 312-324. doi:10.1111/1467-9566.12227

Mead, G. H. (2010). Espíritu, persona y sociedad, desde el punto de vista del conductismo social. Buenos Aires: Editorial Paidós.

MINEDUC. (2009). Ministerio de Educación. Antecedentes generales PIE. Santiago de Chile. Recuperado en https://www.ayudamineduc.cl/ficha/antecedentes-generales-pie-5

. (2013). Orientaciones Técnicas PIE. Santiago de Chile. Recuperado en

https://especial.mineduc.cl > Orientaciones-PIE-2013-3 
(2016). Manual de apoyo a la inclusión escolar en el marco de la reforma educacional. Santiago de Chile. Recuperado en especial.mineduc.cl > Manual-PIE.leyOK_.web_-1.pdf

Mostajo, R. (2000). Gasto social y distribución del ingreso: caracterización e impacto redistributivo en países seleccionados de América Latina y el Caribe. Series Históricas Cepal, 69(7), 69 - 123.

Larrañaga, O. (2007). Qué puede esperarse de la política social en Chile. Disponible en http:// repositorio.uchile.cl/handle/2250/144546

Nina, O. \& Nina, E. (2004). El Efecto Redistributivo de los Impuestos y del Gasto Social Corriente en Bolivia. Development Research Working Paper Series 05/2004, Institute for Advanced Development Studies.

Peña, M. (2017). No estar aquí: Exclusiones en una escuela de estrato socioeconómico bajo de Santiago De Chile. Educação \& Sociedade, 38(140), 751-766.

- (2013). Análisis crítico de discurso del decreto 170 de subvención diferenciada para necesidades educativas especiales: El diagnóstico como herramienta de gestión. Psicoperspectivas, 12(2), 9-103. Doi: 10.5027/psicoperspectivas-Vol12-Issue2-fulltext-252

Peña, M. y Toledo, C. (2017). Discursos sobre clase social y meritocracia de escolares vulnerables en Chile. Cuadernos de Pesquisa, 47(64).

Revuelta, B. y Hernández-Arancibia, R. (2019). La teoría de Axel Honneth sobre justicia social, reconocimiento y experiencias del sujeto en las sociedades contemporáneas. Cinta de moebio, (66), 333-346. https://dx.doi.org/10.4067/s0717-554x2019000300333

Rojas, T. y Armijo, M. (2016). Qué es la inclusión escolar: distintas perspectivas en debate. Cuaderno de Educación, (75), 1-12.

Rojas Fabris, M. T. (2018). Inclusión social: miradas de los docentes y apoderados frente a la mixtura social en sus escuelas. Estudios pedagógicos (Valdivia), 44(3), 217-234.

Tamayo, M., Arrau, M., Sánchez, M., Besoaín, A. y Rebolledo, J. (2018). Programa de Integración Escolar en Chile: brechas y desafíos para la implementación de un programa de educación inclusiva. Revista Española de Discapacidad, 6(1) 161-179. https://doi.org/10.5569/23405104.06.01.08

Thomas, N. (2012). Love, rights and solidarity: Studying children's participation using Honneth's theory of recognition. Childhood, 19(4), 453-466. https://doi.org/10.1177/0907568211434604

Thomas, N., Graham, A., Powell, M. A. \& Fitzgerald, R. (2016). Conceptualisations of children's wellbeing at school: The contribution of recognition theory. Childhood, 23(4), 506-520. https:// doi.org/10.1177/0907568215622802

Winnicott, D. (1981). Los procesos de maduración y el ambiente facilitador. Estudios para una teoría del desarrollo emocional. Barcelona: Editorial Laia. 
